University of Nebraska - Lincoln

DigitalCommons@University of Nebraska - Lincoln

July 1977

\title{
Behavior of partial cross sections and branching ratios in the neighborhood of a resonance
}

Anthony F. Starace

University of Nebraska-Lincoln, astarace1@unl.edu

Follow this and additional works at: https://digitalcommons.unl.edu/physicsstarace

Part of the Physics Commons

Starace, Anthony F., "Behavior of partial cross sections and branching ratios in the neighborhood of a resonance" (1977). Anthony F. Starace Publications. 8.

https://digitalcommons.unl.edu/physicsstarace/8

This Article is brought to you for free and open access by the Research Papers in Physics and Astronomy at DigitalCommons@University of Nebraska - Lincoln. It has been accepted for inclusion in Anthony F. Starace Publications by an authorized administrator of DigitalCommons@University of Nebraska - Lincoln. 


\title{
Behavior of partial cross sections and branching ratios in the neighborhood of a resonance*
}

\author{
Anthony F. Starace ${ }^{\dagger}$ \\ Behlen Laboratory of Physics, The University of Nebraska, Lincoln, Nebraska 68588
}

(Received 3 November 1976; revised manuscript received 4 February 1977)

\begin{abstract}
Starting from the treatment of Fano for the behavior of the total cross section in a photoionization (or electron-ion scattering) experiment in the vicinity of a resonance, we present a theoretical formula for the behavior of an individual final-state channel in the neighborhood of a resonance. This result is then used to derive another theoretical formula for the behavior of the ratio of two partial cross sections (i.e., the branching ratio) in the vicinity of a resonance. This branching-ratio formula depends on the profile parameters $q, \Gamma$, and $\rho^{2}$ for the resonance, on the branching ratio outside the resonance, and on two new parameters which are explicitly related to scattering-matrix elements and phase shifts.
\end{abstract}

\section{INTRODUCTION}

When an atom (or ion) is photoionized, the residual ion may be left possibly in any of a number of term levels, each possibly having a number of fine-structure levels. The relative intensities (i.e., the branching ratios) of the photoelectrons corresponding to these alternative ionic states have been measured recently for the rare gases ${ }^{1}$ and for mercury. ${ }^{2}$ Corresponding measurements have been made for the case of photodissociation of negative ions. 3 All of these experimental studies have found nonstatistical branching ratios for the alternative photoelectron groups and theorists have only begun to explain these results in detail..$^{5-11}$

In this paper we present theoretical expressions for the behavior of partial cross sections and branching ratios within a resonance. These expressions are given in a form analogous to the Fano formula ${ }^{12-14}$ for the profile of an autoionizing resonance. Thus experimental data on branching ratios or partial cross sections within a resonance may be fit in terms of a few parameters using the expressions in this paper. Alternatively, these parameters may be calculated theoretically in terms of electrostatic and spin-orbit interaction matrix elements, as indicated in this paper, and then used to predict the behavior of branching ratios or partial cross sections within a resonance.

A first application of the present theory has been made to recent data of Kemeny et al..$^{15}$ They used synchrotron radiation to scan the $\mathrm{Xe}\left(5 s 5 p^{6} 6 p^{1} P_{1}\right)$ autoionizing resonance centered at $20.95 \mathrm{eV}$ and measured the ${ }^{2} P_{3 / 2}:{ }^{2} P_{1 / 2}$ photoelectron intensity ratio corresponding to the ionization process

$$
\mathrm{Xe}\left(5 p^{6}\right)+\hbar \omega \rightarrow \mathrm{Xe}^{+}\left(5 p^{5}\left({ }^{2} P_{3 / 2,1 / 2}\right)\right)+e^{-} \text {. }
$$

Whereas this ratio had been found previously ${ }^{1}$ to have the nonstatistical value of $\mathbf{1 . 5 5}$ away from any resonances and to be remarkably constant over a range of photon energies of $\simeq 30 \mathrm{eV}$ above the first ionization threshold, Kemeny et al. found ${ }^{15}$ that within the $\operatorname{Xe}\left(5 s 5 p^{6} 6 p^{1} P_{1}\right)$ resonance this ratio increases sharply from its off-resonance value to values greater than 3 . Low signalto-noise ratios prevented an experimental determination of the maximum value of the branching ratio, which occurs near the center of the window resonance. However, a theoretical $\mathrm{fit}^{15}$ to their data using the formulas in this paper indicates that this branching ratio reaches a maximum value of $\simeq 8.8$ within the resonance. This dramatic behavior of the branching ratio is another indication of the strong effects due to electron correlation and spinorbit interaction that can take place in narrow energy regions. Similar dramatic changes have been found in the value of the photoelectron asymmetry parameter within an autoionizing resonance. ${ }^{16,17}$

In Sec. II we review the Fano treatment ${ }^{12}$ of the interaction between an isolated resonance and two or more continua. This results in a description of the behavior of the total cross section in the neighborhood of an isolated resonance. In Sec. III we show how the Fano treatment may be modified to obtain the behavior of partial cross sections in the vicinity of an isolated resonance. In Sec. IV we specialize to the case where the total cross section consists of the sum of two partial cross sections and present a theoretical expression for the branching ratio of the two partial cross sections within a resonance. The procedure in Sec. IV may be extended to three or more partial cross sections, but we have in mind primarily the rare gases and other closed-shell atoms where photoionization of a $p^{6}, d^{10}$, or $f^{14}$ subshell results in two groups of photoelectrons corresponding to the two fine-structure levels of the resulting ion. (It should be noted that although in this paper we have in mind photoionization processes in atoms, the theoretical formulas presented apply equally well 
to photoionization of ions or to inelastic electron scattering from atoms or ions.) Section IV also indicates the relationship between certain parameters appearing in our expression for the branching ratio within a resonance and the Fano profile parameter $\mathrm{s}^{12-14}$ for the resonance. Section V summarizes the main results of this paper for convenient reference. Section VI provides illustrations of the type of behavior one may expect for the branching ratio within an autoionizing resonance, and discusses extensions of the present work and its relation to other work.

While the present theory has been reported earlier ${ }^{18}$ publication of the details of the theory was deferred until an explicit application could be presented and compared to experiment. This goal has now been achieved ${ }^{15,19}$ and the purpose of the present paper is to present the details of the theory reported earlier. ${ }^{15,18,19}$ In the meanwhile, Kabachnik and Sazhina ${ }^{20}$ have developed a similar but somewhat less general theory for describing the behavior of the photoelectron angular distribution and spin polarization within the neighborhood of a resonance. The relation of the present theory to that of Kabachnik and Sazhina ${ }^{20}$ is described in Sec. IV.

\section{REVIEW OF THE FANO TREATMENT FOR THE INTERACTION BETWEEN A SINGLE DISCRETE STATE AND TWO OR MORE CONTINUA}

The Fano treatment ${ }^{12}$ for the interaction between a discrete state and $N$ continuum channels consists in transforming the $N$ degenerate continuum states at a given total energy to $N$ new orthonormal states having only a single member which interacts with the discrete state. This single continuum state belongs to a channel which then interacts with the discrete state just as if the other $N-1$ continuum channels were not there. That is, the $N-1$ other continuum channels merely form a background that is unaffected by the discrete state. We review the transformation to this new set of continuum channels as well as their interaction with the discrete state in this section. While this new set of continuum states is convenient for describing the effect of the discrete state on the total cross section, it is not directly useful however for describing the effect of the discrete state on partial cross sections since the new continuum channels differ from the experimentally observed channels. The relation of the new continuum channels to the experimentally observed channels and the effect of the discrete state on the observed channels is presented in Sec. III.

Following Fano $^{12}$ we start with a discrete state $|\phi\rangle$ and a set of $N$ prediagonalized continuum states $|i \epsilon\rangle$, where $i$ specifies a set of channel quan- tum numbers and $\epsilon$ specifies the total energy of the state. These states are defined to give the following matrix elements of the total Hamiltonian $H$ :

$$
\begin{aligned}
& \left\langle i \epsilon|H| j \epsilon^{\prime}\right\rangle \equiv \epsilon \delta_{i j} \delta\left(\epsilon-\epsilon^{\prime}\right), \\
& \langle\phi|H| \phi\rangle \equiv E_{\phi}, \\
& \langle i \epsilon|H| \phi\rangle \equiv\langle i \epsilon|V| \phi\rangle .
\end{aligned}
$$

The so-called prediagonalized continuum states $|i \epsilon\rangle$, defined by Eq. (1a), get their name from the two-step procedure used in Ref. 12 to diagonalize the Hamiltonian: firstly, the submatrix of the Hamiltonian defined by the (open) continuum channels is diagonalized to obtain the state $|i \epsilon\rangle$; secondly, the interaction of the continuum states $|i \epsilon\rangle$ with the resonance state $|\phi\rangle$ is examined. Reference 12 and the present paper concentrate on the second step of the calculation, the discrete-continuum interaction. Reference 12 assumes the first step has already been carried out; below we indicate the gist of the calculation, since later we shall need various matrices and phase shifts arising from the calculation. However, we refer the reader to Ref. 21 for complete details of the first step of the calculation.

Briefly, then, one starts with a complete set of one-electron orbitals computed from some model Hamiltonian and forms Slater determinants to describe the ionic core and an excited, continuum electron. One can then form linear combinations of these Slater determinants, which diagonalize the ionic core interactions that give rise to different term levels and fine-structure levels. The resulting states, which we shall denote by $|\mu \epsilon\rangle$, are identified by quantum numbers $\mu$ and total energy $\epsilon$, where $\mu$ indicates the fine-structure levels of the core, the orbital angular momentum of the continuum electron, and the coupling of the core and the continuum electron, and where $\epsilon$ is the sum of the core energy and the continuum-electron energy. The states $|\mu \epsilon\rangle$ thus correspond to the asymptotically observable states of the ion-electron system, but they are not eigenstates of the Hamiltonian, i.e., in general, $\left\langle\mu^{\prime} \epsilon^{\prime}|H| \mu \epsilon\right\rangle \neq 0$. If we diagonalize the submatrix of the Hamiltonian defined by the states $|\mu \epsilon\rangle$ (i.e., leaving out interactions with the resonance states), then the standing-wave eigenstates $|i \epsilon\rangle$ of this submatrix-the "prediagonalized" states-may be written as

$$
\begin{aligned}
|i \epsilon\rangle=\sum_{\mu}\left\{|\mu \epsilon\rangle+\sum_{\mu^{\prime}} \mathrm{P} \int d \epsilon^{\prime} \frac{\left|\mu^{\prime} \epsilon^{\prime}\right\rangle\left\langle\mu^{\prime} \epsilon^{\prime}|K(\epsilon)| \mu \epsilon\right\rangle}{\epsilon-\epsilon^{\prime}}\right\} \\
\times T_{\mu i}(\epsilon) \cos \pi \eta_{i},
\end{aligned}
$$

where $\left\langle\mu^{\prime} \epsilon^{\prime}|K(\epsilon)| \mu \epsilon\right\rangle$ is the reaction matrix of collision theory; $\mathrm{P}$ indicates that the Cauchy prin- 
cipal part is taken when integrating over the singularity in the denominator, and the matrix $T_{\mu i}(\epsilon)$ and the phase shifts $\eta_{i}$ are obtained by diagonalizing the on-the-energy-shell reaction matrix according to the following equation:

$$
\sum_{\mu^{\prime}}\left\langle\mu \epsilon|K(\epsilon)| \mu^{\prime} \epsilon\right\rangle T_{\mu^{\prime} i}(\epsilon)=-\pi^{-1} \tan \pi \eta_{i} T_{\mu i}(\epsilon) .
$$

The reaction matrix is obtained by the standard methods of continuum perturbation theory. ${ }^{21}$ Note that the eigenstates $|i \epsilon\rangle$ do not refer to any particular core energy or photoelectron energy but rather to a superposition of observable states as indicated in Eq. (1d).

We now represent an exact standing-wave finalstate eigenfunction of the total Hamiltonian $H$ as the following superposition of the states $|\phi\rangle$ and $|i \epsilon\rangle$ :

$$
\mid \lambda E)=a(\lambda E)|\phi\rangle+\sum_{i=1}^{N} \int d \epsilon b_{i \epsilon}(\lambda E)|i \epsilon\rangle .
$$

The index $\lambda$ represents the set of quantum numbers necessary to specify the new channel, $E$ is the total energy of the state, and we use rounded bras and kets to distinguish the eigenvectors of the total Hamiltonian.

Taking matrix elements of $H$ between the ket $\mid \lambda E)$ on the right and either $\langle\phi|$ or $\left\langle j \epsilon^{\prime}\right|$ on the left, we obtain the following linear equations for the coefficients $a(\lambda E)$ and $b_{i \in}(\lambda E)$ :

$$
\begin{aligned}
& a(\lambda E) E_{\phi}+\sum_{i=1}^{N} \int d \epsilon b_{i \epsilon}(\lambda E)\langle\phi|V| i \epsilon\rangle=E a(\lambda E) \\
& a(\lambda E)\left\langle j \epsilon^{\prime}|V| \phi\right\rangle+b_{j \epsilon^{\prime}}(\lambda E) \epsilon^{\prime}=E b_{j \epsilon^{\prime}}(\lambda E)
\end{aligned}
$$

Equations (3a) and (3b) describe the interaction of $|\phi\rangle$ with the $N$ prediagonalized continuum states $|i \epsilon\rangle$. For fixed total energy $\epsilon$ the $N$ states $|i \epsilon\rangle$ are degenerate, and hence we may make linear combinations of them to form any other complete set of $N$ continuum states. 'In particular, we wish to form linear combinations of the states $|i \epsilon\rangle$ so that only one of the new basis states interacts with $|\phi\rangle$ and the rest are orthogonal to it and hence unperturbed.

Noticing that Eq. (3a) involves the sum

$$
S_{\epsilon}(\lambda E) \equiv \sum_{i=1}^{N}\langle\phi|V| i \epsilon\rangle b_{i \epsilon}(\lambda E),
$$

we rewrite Eq. (3a) in terms of $S_{\epsilon}(\lambda E)$ and then multiply Eq. (3b) by $\left\langle\phi|V| j \epsilon^{\prime}\right\rangle$ and sum over $j$ to get the following pair of equations:

$$
\begin{aligned}
& a(\lambda E) E_{\phi^{+}} \int d \epsilon S_{\epsilon}(\lambda E)=E a(\lambda E), \\
& a(\lambda E) \frac{\Gamma(\epsilon)}{2 \pi}+\epsilon S_{\epsilon}(\lambda E)=E S_{\epsilon}(\lambda E) .
\end{aligned}
$$

In Eq. (5b) we have introduced the linewidth, which is defined as

$$
\Gamma(\epsilon) \equiv 2 \pi \sum_{i=1}^{N}|\langle i \epsilon|V| \phi\rangle|^{2} .
$$

Equations (5a) and (5b) describe the interaction of the discrete state $|\phi\rangle$ with the linear combination $S_{\epsilon}(\lambda E)$ of the continuum states $|i \epsilon\rangle$. We have thus rewritten Eq. (3a) as Eq. (5a) and taken a particular linear combination of the $N$ equations (i.e., $1 \leq j \leq N$ ) represented by Eq. (3b). To complete our transformation of Eqs. (3a) and (3b), we form $N-1$ other linear combinations of the $N$ equations represented by Eq. (3b) and choose these $N-1$ other equations in such a way that there is no coupling to the discrete state $|\phi\rangle$. The $k$ th member of these $N-1$ linear combinations is formed by multiplying Eq. (3b) for $j=1$ by $\left\langle k \epsilon^{\prime}|V| \phi\right\rangle$ and subtracting the result from the result of multiplying Eq. (3b) for $j=k$ by $\left\langle 1 \epsilon^{\prime}|V| \phi\right\rangle$. One obtains the $N-1$ equations $(2 \leq k \leq N)$ :

$$
\left(E-\epsilon^{\prime}\right)\left[b_{k \epsilon^{\prime}}(\lambda E)\left\langle 1 \epsilon^{\prime}|V| \phi\right\rangle-b_{1 \epsilon^{\prime}}(\lambda E)\left\langle k \epsilon^{\prime}|V| \phi\right\rangle\right]=0 \text {. }
$$

Rather than solving Eqs. (3a) and (3b) for the coefficients $a(\lambda E)$ and $b_{i \epsilon}(\lambda E)$, we will thus solve Eqs. (5a), (5b); and (5d) for these coefficients. As discussed, we are looking for two kinds of eigenvectors $\mid \lambda E$ ) in Eq. (2): one which has a discrete component [i.e., $a(\lambda E) \neq 0$ for $\lambda=1$ ] and $N-1$ others which do not [i.e., $a(\lambda E)=0$ for $2 \leq \lambda \leq N]$. We consider each of these two cases in turn.

If $a(\lambda E) \neq 0$, then Eqs. (5a) and (5b) are of the same form as the equations describing the interaction of a single discrete state with a single continuum channel; these equations thus have the solutions $\mathrm{s}^{12}$

$$
\begin{aligned}
S_{\epsilon}(1 E) \equiv & \sum_{j=1}^{N}\langle\phi|V| j \epsilon\rangle b_{j \epsilon}(1 E) \\
= & \left(\frac{P}{E-\epsilon} \frac{\sin \Delta(E)}{\pi}-\delta(E-\epsilon) \cos \Delta(E)\right) \\
& \times \frac{\Gamma(\epsilon)}{[2 \pi \Gamma(E)]^{1 / 2}}, \\
a(1 E)= & \left(\frac{2 \pi}{\Gamma(E)}\right)^{1 / 2} \frac{\sin \Delta(E)}{\pi},
\end{aligned}
$$

where

$$
\tan \Delta(E) \equiv \frac{-\frac{1}{2} \Gamma(E)}{E-E_{\phi}-(P / 2 \pi) \int \Gamma(\epsilon) d \epsilon /(E-\epsilon)} .
$$

In Eq. (6) the symbol $P$ indicates that the Cauchy principal part is to be taken in any integration 
over energy $\epsilon$ and that $\lambda$ has been set equal to 1 . Comparing Eq. (6a) and Eq. (5d) and using the definition in Eq. (5c), we see that the coefficients $b_{j \epsilon}(1 E)$ are given by

$$
\begin{aligned}
b_{j \epsilon}(1 E)= & \left(\frac{2 \pi}{\Gamma(E)}\right)^{1 / 2} \\
& \times\left(\frac{P}{E-\epsilon} \frac{\sin \Delta(E)}{\pi}-\delta(E-\epsilon) \cos \Delta(E)\right) \\
& \times\langle j \epsilon|V| \phi\rangle .
\end{aligned}
$$

Substituting Eqs. (6b) and (6d) in Eq. (2) we obtain for the $\lambda=1$ eigenvector the following:

$$
\begin{aligned}
\mid 1 E)= & \left(\frac{2 \pi}{\Gamma(E)}\right)^{1 / 2} \\
& \times\left(|\Phi\rangle \frac{\sin \Delta(E)}{\pi}-\sum_{i=1}^{N}|i E\rangle\langle i E|V| \phi\rangle \cos \Delta(E)\right) .
\end{aligned}
$$

In Eq. (7) we have defined the "augmented discrete state" $\Phi$ as $^{12}$

$$
|\Phi\rangle \equiv|\phi\rangle+\sum_{i=1}^{N} P \int d \epsilon \frac{|i \epsilon\rangle\langle i \epsilon|V| \phi\rangle}{E-\epsilon}
$$

Note that Eq. (7) exhibits resonant behavior through functions of the angle $\Delta(E)$, which is defined in Eq. (6c).

The $N-1$ other solutions $(2 \leq \lambda \leq N)$ are obtained by setting $a(\lambda E)=0$ in Eqs. (5a) and (5b), which implies that

$$
S_{\epsilon}(\lambda E) \equiv \sum_{j=1}^{N}\langle\phi|V| j \epsilon\rangle b_{j \epsilon}(\lambda E)=0,
$$

and then requiring that the coefficients $b_{j \epsilon}(\lambda E)$ satisfy both Eq. (5d) and Eq. (9). If we require each $b_{j \epsilon}(\lambda E)$ to be proportional to $\delta(E-\epsilon)$, then Eq. (5d) is satisfied and we are left with finding the $N-1$ orthonormal solutions of Eq. (9). [Note that Eq. (9) represents a homogeneous equation in $N$ unknowns having a coefficient matrix of rank 1; therefore the solutions span a vector space of $N-1$ dimensions. ${ }^{22}$ ] A set of $N-1$ linearly independent solutions of Eq. (9), all orthogonal to the $\lambda=1$ solution, may be found quite easily. For example, one might define the $\lambda$ th solution $(2 \leq \lambda \leq N)$ as follows:

$$
\begin{aligned}
& b_{i \epsilon}(\lambda E)=-\delta(E-\epsilon)\langle\phi|V| \lambda \epsilon\rangle, \\
& b_{\lambda \epsilon}(\lambda E)=\delta(E-\epsilon)\langle\phi|V| 1 \epsilon\rangle, \\
& b_{j \epsilon}(\lambda E)=0 \text { for } j \neq 1 \text { and } j \neq \lambda .
\end{aligned}
$$

The coefficients for these $N-1$ solutions, when substituted in Eq. (2), give $N-1$ continuum eigenvectors having no coupling to the discrete state and, of course, no resonant behavior. They may be made orthonormal to each other by the Gram-
Schmidt orthogonalization procedure.

In summary, the eigenvectors in Eq. (2) may be written for $\lambda=1$ and for $2 \leq \lambda \leq N$, respectively, as follows:

$$
\begin{aligned}
\mid 1 E)= & |\Phi\rangle\left(\frac{2 \pi}{\Gamma(E)}\right)^{1 / 2} \frac{\sin \Delta(E)}{\pi} \\
& +\sum_{i=1}^{N}|i E\rangle U_{i 1}(E) \cos \Delta(E), \\
\mid \lambda E)= & \sum_{i=1}^{N}|i E\rangle U_{i \lambda}(E) .
\end{aligned}
$$

In Eq. (11) the unitary transformation matrix that transforms the prediagonalized states $|i E\rangle$ into the new linear combinations

$$
\sum_{i=1}^{N}|i E\rangle U_{i \lambda}(E)
$$

has the following properties. Firstly, for $2 \leq \lambda \leq N$ it transforms the states $|i E\rangle$ into eigenvectors $\mid \lambda E$ ) which have no interaction with the discrete state. The specific form of $U_{i \lambda}(E)$ for $2 \leq \lambda \leq N$ depends on the number of channels $N$ as indicated in the discussion following Eq. (10). Secondly, for $\lambda=1$ we have

$$
U_{i 1}(E)=-\left(\frac{2 \pi}{\Gamma(E)}\right)^{1 / 2}\langle i E|V| \phi\rangle
$$

as may be seen by comparison of Eq. (11a) with Eq. (7). The extent of the mixing of the linear combination

$$
\sum_{i=1}^{N}|i E\rangle U_{i 1}(E)
$$

with the augmented discrete state is determined by the magnitude of the angle $\Delta(E)$, which is defined in Eq. (6c).

The meaning of the angle $\Delta(E)$ may be found by rewriting Eq. (11a) as follows. Factor out $\cos \Delta(E)$ from Eq. (11a) and replace $\tan \Delta(E)$ by

$$
\frac{\tan \Delta(E)}{\pi}=-\sum_{i=1}^{N} \frac{\langle\phi|V| i E\rangle\langle i E|V| \phi\rangle}{E-E_{\phi}-(P / 2 \pi) \int \Gamma(\epsilon) d \epsilon /(E-\epsilon)},
$$

which follows from Eqs. (5c) and (6c). Then, using Eq. (12), one obtains

$$
\begin{aligned}
\mid 1 E)=\sum_{i=1}^{N} & \left(\frac{|\Phi\rangle\langle\phi|V| i E\rangle}{E-E_{\phi}-(P / 2 \pi) \int \Gamma(\epsilon) d \epsilon /(E-\epsilon)}+|i E\rangle\right) \\
& \times U_{i_{1}}(E) \cos \Delta(E) .
\end{aligned}
$$

Equation (14) shows that $\Delta(E)$ is the phase shift in the eigenchannel $\lambda=1$ due to interaction of the states $|i E\rangle$ with the isolated resonance state. ${ }^{21}$ Equation (11b) correspondingly shows that there is no such phase shift in the eigenchannels $\lambda>1$. 


\section{BEHAVIOR OF PARTIAL CROSS SECTIONS IN THE VICINITY OF AN ISOLATED RESONANCE}

In the previous section, following Fano's treatment ${ }^{12}$ we obtained standing-wave expressions in Eqs. (11b) and (14) for the eigenfunctions of the total Hamiltonian. Only one of these eigenfunctions, denoted by $\lambda=1$, exhibits the characteristic resonance behavior associated with an autoionizing state. While this choice of eigenstates $\lambda$ simplifies the description of the discrete-continuum interaction, it does not permit a simple relation to experimentally measured final states, which most often are not eigenstates of the collision process, but rather states having a well-defined energy for the residual ion and a well-defined kinetic energy for the outgoing electron. In other words, the eigenchannels $\lambda$ do not satisfy the final-state boundary condition that there is an outgoing electron in a particular observable channel $\mu$, characterized by the state of the ion and its coupling with the outgoing electron and by well-defined ionic and electronic energies.

We may obtain the desired final state ket of total energy $E$ as a linear combination of the standing-wave eigenfunctions $\mid \lambda E)$,

$$
\left.|\mu E-\rangle=\sum_{\lambda=1}^{N} \mid \lambda E\right) \chi_{\lambda}^{(\mu E-)},
$$

where the minus sign indicates that the coefficients $\chi_{\lambda}^{(\mu E-)}$ are determined by applying the so-called incoming-wave boundary condition for the channel $\mu$. This boundary condition requires that asymptotically (i.e., as $r \rightarrow \infty$ ) the amplitudes for all outgoing spherical waves in channels other than $\mu$ vanish. Application of this boundary condition gives $^{23}$

$$
\chi_{\lambda}^{(\mu E-)}=\exp \left(-i \Delta_{\lambda}\right) \sum_{i=1}^{N} \tilde{U}_{\lambda i}(E) \exp \left(-i \eta_{i}\right) \tilde{T}_{i \mu}(E),
$$

where the orthogonal matrix $T_{\mu i}(E)$, defined in Eq. (1e), transforms the experimentally observable channels $\mu$ at energy $E$ to the prediagonalized continuum channels $i$; the phase shift $\eta_{i}$, also defined in Eq. (1e), is the eigenphase shift in the channel $i$ arising from the interaction between different channels $\mu$; the matrix $U_{i \lambda}(E)$, defined in the previous section, transforms the prediagonalized states $i$ to the eigenstates $\lambda$; and $\Delta_{\lambda}=\Delta \delta_{\lambda_{1}}$ is the eigenphase shift in the channel $\lambda$ arising from the interaction of the channels $i$ with the discrete resonance state. Note that the states $\left|\mu E_{-}\right\rangle$in Eq. (15) are the incoming-wave-normalized counterparts of the standing-wave-normalized states $|\mu E\rangle$ in Eqs. (1d) and (1e).

The experimentally observable final states $|\mu E-\rangle$ are thus completely defined by Eqs. (15), (11b), (14), and (16). We consider now the matrix element for an electric dipole transition to one of these final states from an initial state indicated by $\left\langle\psi_{0}\right|$. We obtain a convenient form for this matrix element by first substituting Eqs. (11b), (14), and (16) in Eq. (15) and defining the following expression for compactness:

$$
\Omega_{\lambda \mu}(E) \equiv \sum_{i=1}^{N} \tilde{U}_{\lambda i}(E) \exp \left(-i \eta_{i}\right) \tilde{T}_{i \mu}(E) .
$$

In this way we find

$$
\begin{aligned}
\left\langle\psi_{0}|\overrightarrow{\mathrm{r}}| \mu E-\right\rangle= & \sum_{\lambda=2}^{N} \sum_{i=1}^{N}\left\langle\psi_{0}|\overrightarrow{\mathrm{r}}| i E\right\rangle U_{i \lambda}(E) \Omega_{\lambda \mu}(E) \\
& +\sum_{i=1}^{N}\left(\frac{\left\langle\psi_{0}|\overrightarrow{\mathrm{r}}| \Phi\right\rangle\langle\phi|V| i E\rangle}{E-E_{\phi}-(\mathrm{P} / 2 \pi) \int \Gamma(\epsilon) d \epsilon /(E-\epsilon)}+\left\langle\psi_{0}|\overrightarrow{\mathrm{r}}| i E\right\rangle\right) U_{i 1}(E) \Omega_{1 \mu} \cos \Delta e^{-i \Delta} .
\end{aligned}
$$

Secondly we note that far from the resonance, or in the absence of the resonance, $\Delta \rightarrow 0$ and also

$$
\frac{\left\langle\psi_{0}|\vec{r}| \Phi\right\rangle\langle\phi|V| i E\rangle}{E-E_{\phi}-(\mathrm{P} / 2 \pi) \int \Gamma(\epsilon) d \epsilon /(E-\epsilon)} \rightarrow 0 .
$$

Hence we may write the matrix element in the absence of the resonance as

$$
\left\langle\psi_{0}|\overrightarrow{\mathbf{r}}| \mu E-\right\rangle_{0} \equiv \sum_{\lambda=1}^{N} \sum_{i=1}^{N}\left\langle\psi_{0}|\overrightarrow{\mathbf{r}}| i E\right\rangle U_{i \lambda}(E) \Omega_{\lambda \mu}(E) .
$$

Substituting Eq. (20) in Eq. (18) we get

$$
\begin{aligned}
\frac{\left\langle\psi_{0}|\overrightarrow{\mathbf{r}}| \mu E-\right\rangle}{\left\langle\psi_{0}|\overrightarrow{\mathrm{r}}| \mu E-\right\rangle_{0}}=1+\left\{\frac{\left\langle\psi_{0}\left|\overrightarrow{\mathbf{r}}_{i}\right| \Phi\right\rangle}{\left\langle\psi_{0}|\overrightarrow{\mathrm{r}}| \mu E-\right\rangle_{0}} \frac{\sum_{i=1}^{N}\langle\phi|V| i E\rangle U_{i 1}(E) \Omega_{1 \mu}(E)}{E-E_{\phi}-(\mathrm{P} / 2 \pi) \int \Gamma(\epsilon) d \epsilon /(E-\epsilon)}\right. \\
\left.+\frac{\sum_{i=1}^{N}\left\langle\psi_{0}|\overrightarrow{\mathbf{r}}| i E\right\rangle U_{i 1}(E) \Omega_{1 \mu}(E)}{\left\langle\psi_{0}|\overrightarrow{\mathbf{r}}| \mu E-\right\rangle_{0}}\left(1-\frac{\exp (i \Delta)}{\cos \Delta}\right)\right\} \cos \Delta \exp (-i \Delta) .
\end{aligned}
$$


Equation (21) may now be written in an especially simple form by (i) writing $\left\langle\psi_{0}|\overrightarrow{\mathrm{r}}| \Phi\right\rangle$ in terms of the Fano $q$ parameter: ${ }^{24}$

$$
\left\langle\psi_{0}|\overrightarrow{\mathrm{r}}| \Phi\right\rangle=\pi q \sum_{i=1}^{N}\left\langle\psi_{0}|\overrightarrow{\mathrm{r}}| i E\right\rangle\langle i E|V| \phi\rangle,
$$

(ii) writing $\Delta(E)$ and the energy denominator in Eq. (21) in terms of the reduced energy variable $\epsilon:^{12}$

$$
\epsilon \equiv \frac{E-E_{\phi}-(\mathrm{P} / 2 \pi) \int \Gamma(\epsilon) d \epsilon /(E-\epsilon)}{\frac{1}{2} \Gamma(\epsilon)}=-\cot \Delta(E),
$$

(iii) introducing the new variable $\alpha(\mu E)$ :

$$
\begin{aligned}
\alpha(\mu E) & \equiv \frac{\sum_{i=1}^{N}\left\langle\psi_{0}|\dot{\vec{r}}| i E\right\rangle U_{i 1}(E) \Omega_{1 \mu}(E)}{\left\langle\psi_{0}|\overrightarrow{\mathrm{r}}| \mu E-\right\rangle_{0}} \\
& =\frac{2 \pi}{\Gamma(\epsilon)} \frac{\left(\sum_{i=1}^{N}\left\langle\psi_{0}|\overrightarrow{\mathrm{r}}| i E\right\rangle\langle i E|V| \phi\rangle\right)\left[\sum_{j=1}^{N}\langle\phi|V| j E\rangle \exp \left(-i \eta_{j}\right) \tilde{T}_{j \mu}(E)\right]}{\left\langle\psi_{0}|\overrightarrow{\mathrm{r}}| \mu E-\right\rangle_{0}},
\end{aligned}
$$

and (iv) substituting Eqs. (5c), (12), (17), and (22)-(24) in Eq. (21) to obtain

$$
\left\langle\psi_{0}|\overrightarrow{\mathbf{r}}| \mu E-\right\rangle=\left\langle\psi_{0}|\overrightarrow{\mathrm{r}}| \mu E-\right\rangle_{0}[1+\alpha(\mu E)(q+i) /(\epsilon-i)] .
$$

Finally, noting from Eq. (24) that $\alpha(\mu E)$ is complex and thus writing $\alpha(\mu E)=\operatorname{Re} \alpha(\mu E)+i \operatorname{Im} \alpha(\mu E)$, we obtain for the squared dipole-matrix element the following expression:

$$
\begin{aligned}
\left|\left\langle\psi_{0}|\overrightarrow{\mathrm{r}}| \mu E-\right\rangle\right|^{2}= & \frac{\left|\left\langle\psi_{0}|\overrightarrow{\mathrm{r}}| \mu E-\right\rangle_{0}\right|^{2}}{1+\epsilon^{2}} \\
& \times\left\{\epsilon^{2}+2 \epsilon[q \operatorname{Re} \alpha(\mu E)-\operatorname{Im} \alpha(\mu E)]+\left[1-2 q \operatorname{Im} \alpha(\mu E)-2 \operatorname{Re} \alpha(\mu E)+\left(q^{2}+1\right)|\alpha(\mu E)|^{2}\right]\right\} .
\end{aligned}
$$

Equations (25) and (26) thus give the dipole-matrix element and its absolute square for transition to an observable channel $\mu$ in the vicinity of an autoionizing resonance in terms of the Fano $q$ parameter and the real and imaginary parts of a new parameter $\alpha(\mu E)$. The new parameter $\alpha(\mu E)$, defined by Eq. (24), may be interpreted by comparing Eqs. (20) and (24): It represents the fraction of the dipole amplitude $\left\langle\psi_{0}|\overrightarrow{\mathbf{r}}| \mu E-\right\rangle_{0}$ that passes through the eigenchannel $\lambda=1$, the only eigenchannel which interacts with the discrete state. In the limit of a single channel the matrices in Eq. (24) collapse to unity, as does $\alpha(\mu E)$, and hence Eq. (26) reduces to the Fano profile formula,

$$
\left|\left\langle\psi_{0}|\overrightarrow{\mathbf{r}}| \mu E-\right\rangle\right|^{2}=\left|\left\langle\psi_{0}|\overrightarrow{\mathbf{r}}| \mu E_{-}\right\rangle_{0}\right|^{2} \frac{(q+\epsilon)^{2}}{1+\epsilon^{2}} .
$$

Since $\alpha(\mu E),\left\langle\psi_{0}|\overrightarrow{\mathbf{r}}| \mu E-\right\rangle_{0}$ and $q$ contain no factors having resonant behavior, that is, dependent on $\epsilon$, we expect that in the narrow energy region of a resonance these parameters will be nearly constant [cf. Eqs. (20), (22), and (24)]. That is, the entire energy dependence of the dipole-matrix element and its square in Eqs. (25) and (26) comes from the reduced energy variable $\epsilon$. Hence, given the parameters $\alpha(\mu E),\left\langle\psi_{0}|\overrightarrow{\mathbf{r}}| \mu E-\right\rangle_{0}$, and $q$, one may determine the behavior of any kind of photoabsorption process within an autoionizing resonance. In the following sections we apply Eq. (26) to the determination of the behavior of photoelectron branching ratios within a resonance.

\section{BEHAVIOR OF THE BRANCHING RATIO ACROSS AN ISOLATED RESONANCE}

In the last section we obtained the squared electric-dipole matrix element for transition from the ground state to a particular experimentally observable final state. In many experiments, e.g., photoelectron spectroscopy, what is measured is the branching ratio of different groups of photoelectrons. Consider the case of photoionization of an $n p^{6}$ or $n d^{10}$ subshell of a closed-shell atom or ion. The residual atomic or ionic core after ionization will have two fine-structure levels of different energy [i.e., $n p^{5}\left({ }^{2} P_{3 / 2,1 / 2}\right)$ or $\left.n d^{9}\left({ }^{2} D_{5 / 2,3 / 2}\right)\right]$. For a fixed photon energy, photoelectron spectroscopy experiments thus measure two groups of photoelectrons having different energies corresponding to the two energies of the residual core. The higher-energy group of photoelectrons (corresponding to the higher-multiplicity core state) comprises three observable final-state channels, while the lower-energy group of photoelectrons (corresponding to the lower-multiplicity core state) comprises two observable final-state channels. In this section we shall examine how the ratio of these two photoelectron groups behaves 
in the vicinity of an isolated resonance. A first application of the theory presented in this section has been reported elsewhere. ${ }^{15,19}$

We consider then a group of $N$ final-state channels of which a subset $P$ all have one photoelectron energy and a subset $Q$ have another photoelectron energy. The number of channels belonging to $P$ plus the number of channels belonging to $Q$ equals $N$. The measured cross-section branching ratio will thus be the ratio of the sum of the squared dipole-matrix elements belonging to the individual channels of subset $P$ to the sum of the squared dipole-matrix elements belonging to the individual channels of subset $Q$. That is,

$$
\frac{\sigma_{P}}{\sigma_{Q}}=\frac{\sum_{\mu \in P}\left|\left\langle\psi_{0}|\overrightarrow{\mathrm{r}}| \mu E-\right\rangle\right|^{2}}{\sum_{\mu \in Q}\left|\left\langle\psi_{0}|\overrightarrow{\mathrm{r}}| \mu E-\right\rangle\right|^{2}},
$$

where the individual squared dipole-matrix elements are given by Eq. (26). Note that the photoionization cross section for each channel $\mu$ is equal to the squared dipole-matrix element times a factor involving the photon energy and various fundamental constants. ${ }^{25}$ This factor cancels in numerator and denominator when we take the ratio of the partial cross sections $\sigma_{P}$ and $\sigma_{Q}$.

Substituting Eq. (26) into Eq. (27) we obtain

$$
\frac{\sigma_{P}}{\sigma_{Q}}=\left(\frac{\sigma_{P}}{\sigma_{Q}}\right)_{0}\left(\frac{\epsilon^{2}+2 \epsilon\left(q \operatorname{Re}\langle\alpha\rangle_{P}-\operatorname{Im}\langle\alpha\rangle_{P}\right)+\left[1-2 q \operatorname{Im}\langle\alpha\rangle_{P}-2 \operatorname{Re}\langle\alpha\rangle_{P}+\left(q^{2}+1\right)\left\langle|\alpha|^{2}\right\rangle_{P}\right]}{\epsilon^{2}+2 \epsilon\left(q \operatorname{Re}\langle\alpha\rangle_{Q}-\operatorname{Im}\langle\alpha\rangle_{Q}\right)+\left[1-2 q \operatorname{Im}\langle\alpha\rangle_{Q}-2 \operatorname{Re}\langle\alpha\rangle_{Q}+\left(q^{2}+1\right)\left\langle|\alpha|^{2}\right\rangle_{Q}\right]}\right),
$$

where we have defined the branching ratio away from the resonance as

$$
\left(\frac{\sigma_{P}}{\sigma_{Q}}\right)_{0} \equiv \frac{\sum_{\mu \in P}\left|\left\langle\psi_{0}|\overrightarrow{\mathrm{r}}| \mu E-\right\rangle_{0}\right|^{2}}{\sum_{\mu \in Q}\left|\left\langle\psi_{0}|\overrightarrow{\mathrm{r}}| \mu E-\right\rangle_{0}\right|^{2}},
$$

and weighted averages of the $\alpha(\mu E)$ parameters as

$$
\langle\alpha\rangle_{P} \equiv \frac{\sum_{\mu \in P} \alpha(\mu E)\left|,\left\langle\psi_{0}|\overrightarrow{\mathbf{r}}| \mu E-\right\rangle_{0}\right|^{2}}{\sum_{\mu \in P}\left|\left\langle\psi_{0}|\overrightarrow{\mathbf{r}}| \mu E-\right\rangle_{0}\right|^{2}}
$$

and

$$
\left\langle|\alpha|^{2}\right\rangle_{P} \equiv \frac{\sum_{\mu \in P}|\alpha(\mu E)|^{2}\left|\left\langle\psi_{0}|\overrightarrow{\mathbf{r}}| \mu E-\right\rangle_{0}\right|^{2}}{\sum_{\mu \in P}\left|\left\langle\psi_{0}|\overrightarrow{\mathbf{r}}| \mu E-\right\rangle_{0}\right|^{2}}
$$

The parameters $\langle\alpha\rangle_{Q}$ and $\left\langle|\alpha|^{2}\right\rangle_{Q}$ have sums over $\mu$ belonging to the set $Q$ but are otherwise identical to the definitions in Eqs. (30) and (31).

Equation (28) thus gives the branching ratio in the neighborhood of a resonance as the product of the branching. ratio outside the resonance and an $\epsilon$-dependent factor consisting of the ratio of two quadratic polynomials in $\epsilon$. The coefficients of these polynomials depend on the following six new parameters: $\left\langle|\alpha|{ }^{2}\right\rangle_{P},\left\langle|\alpha|^{2}\right\rangle_{Q}$, and the real and imaginary parts of $\langle\alpha\rangle_{P}$ and $\langle\alpha\rangle_{Q}$. The remainder of this section is devoted to an examination of the properties of these six parameters. In particular, we shall see that Eq. (28) involves only two independent parameters.

\section{A. Relations between the $\alpha$ parameters}

In order to determine the properties of the parameters, we must examine Eqs. (30) and (31) in detail. Substituting Eq. (24) into Eq. (31) and making use of the relation ${ }^{26}$

$$
\begin{aligned}
& \left(\sum_{i=1}^{N}\left\langle\psi_{0}|\overrightarrow{\mathrm{r}}| i E\right\rangle\langle i E|V| \phi\rangle\right)^{2} \\
& =\rho^{2} \frac{\Gamma(E)}{2 \pi} \sum_{\mu=1}^{N}\left|\left\langle\psi_{0}|\overrightarrow{\mathrm{r}}| \mu E_{-}\right\rangle\right|^{2},
\end{aligned}
$$

we obtain

$$
\left\langle|\alpha|^{2}\right\rangle_{\hat{P}}=\rho^{2}\left[1+\left(\sigma_{P} / \sigma_{Q}\right)_{0}^{-1}\right] \chi .
$$

In Eq. (33), $\rho^{2}$ is another profile parameter, called the correlation index ${ }^{26}$ having values $\rho^{2} \leq 1$. The branching ratio outside the resonance, $\left(\sigma_{P} / \sigma_{Q}\right)_{0}$, is given by Eq. (29), and $\chi$ is defined as

$$
\chi \equiv \frac{\sum_{\mu \in P}\left|\sum_{j=1}^{N}\langle\phi|V| j E\rangle \exp \left(-i \eta_{j}\right) \tilde{T}_{j \mu}(E)\right|^{2}}{\sum_{j=1}^{N}|\langle\phi|V| j E\rangle|^{2}} .
$$

Note that in Eq. (34), if the sum over $\mu$ were over the full set of $N$ channels, then $\chi$ would become equal to unity because $T_{\mu j}(E)$ is a unitary matrix. In general, then, $0 \leq \chi \leq 1$. Considerations similar to those which led to Eq. (33) give the additional result

$$
\left\langle|\alpha|^{2}\right\rangle_{Q}=\rho^{2}\left[1+\left(\sigma_{P} / \sigma_{Q}\right)_{0}\right](1-\chi) .
$$

Finally, multiplying Eq. (33) by $\left(\sigma_{p} / \sigma_{Q}\right)_{0}$ and adding the result to Eq. (35) gives a relationship between $\left\langle|\alpha|^{2}\right\rangle_{P}$ and $\left\langle|\alpha|^{2}\right\rangle_{Q}$ :

$$
\left(\sigma_{P} / \sigma_{Q}\right)_{0}\left\langle|\alpha|^{2}\right\rangle_{P}+\left\langle|\alpha|^{2}\right\rangle_{Q}=\rho^{2}\left[1+\left(\sigma_{P} / \sigma_{Q}\right)_{0}\right] \text {. }
$$

Turning now to $\langle\alpha\rangle_{p}$, substitute Eq. (24) in Eq. (30) and make use of Eq. (32) to obtain

$$
\langle\alpha\rangle_{P}=\rho^{2}\left[1+\left(\sigma_{P} / \sigma_{Q}\right)_{0}^{-1}\right] \xi,
$$

where $\xi$ is a complex coefficient defined by 


$$
\xi \equiv \frac{\sum_{\mu \in P}\left[\sum_{j=1}^{N}\langle\phi|V| j E\rangle \exp \left(-i \eta_{j}\right) \tilde{T}_{j \mu}(E)\right]\left\langle\mu E-|\overrightarrow{\mathbf{r}}| \psi_{0}\right\rangle}{\sum_{i=1}^{N}\langle\phi|V| i E\rangle\left\langle i E|\overrightarrow{\mathbf{r}}| \psi_{0}\right\rangle} .
$$

[From Eqs. (17) and (20) and the properties of the unitary matrices $U_{i \lambda}(E)$ and $T_{\mu i}(E)$, we see that if the sum over $\mu$ in Eq. (38) extended over all $N$ channels then $\xi$ would become unity.] A similar calculation shows that

$$
\langle\alpha\rangle_{Q}=\rho^{2}\left[1+\left(\sigma_{P} / \sigma_{Q}\right)_{0}\right](1-\xi) .
$$

Furthermore, multiplying Eq. (37) by $\left(\sigma_{P} / \sigma_{Q}\right)_{0}$ and adding the result to Eq. (39) gives the following relationship:

$$
\left(\sigma_{P} / \sigma_{Q}\right)_{0}\langle\alpha\rangle_{P}+\langle\alpha\rangle_{Q}=\rho^{2}\left[1+\left(\sigma_{P} / \sigma_{Q}\right)_{0}\right] .
$$

Taking the real and imaginary parts of Eq. (40) and noting that the right-hand side is real gives the following two relations:

$$
\begin{aligned}
& \left(\sigma_{P} / \sigma_{Q}\right)_{0} \operatorname{Re}\langle\alpha\rangle_{P}+\operatorname{Re}\langle\alpha\rangle_{Q}=\rho^{2}\left[1+\left(\sigma_{P} / \sigma_{Q}\right)_{0}\right], \\
& \left(\sigma_{P} / \sigma_{Q}\right)_{0} \operatorname{Im}\langle\alpha\rangle_{P}+\operatorname{Im}\langle\alpha\rangle_{Q}=0 .
\end{aligned}
$$

Equations (36), (41), and (42) thus give three equations relating the $\operatorname{six} \alpha$ parameters. Hence if one knows from other experimental measurements the profile parameters $q, \rho^{2}$, and $\Gamma$ for a resonance, as well as the branching ratio outside the resonance, $\left(\sigma_{P} / \sigma_{Q}\right)_{0}$, then one may calculate the branching ratio within the resonance by calculating only the quantities $\left\langle|\alpha|{ }^{2}\right\rangle_{P}, \operatorname{Re}\langle\alpha\rangle_{P}$, and $\operatorname{Im}\langle\alpha\rangle_{P}$. Unfortunately one cannot obtain these $\alpha$ parameters by fitting Eq. (28) to experimental data since after substituting for $\left\langle|\alpha|^{2}\right\rangle_{Q}, \operatorname{Re}\langle\alpha\rangle_{Q}$, and $\operatorname{Im}\langle\alpha\rangle_{Q}$ from Eqs. (36), (41), and (42) in Eq. (28), Eq. (28) has the form

$$
\left(\frac{\sigma_{P}}{\sigma_{Q}}\right)=r\left\{\frac{\epsilon^{2}+2 \epsilon C_{1}+\left(1+C_{2}\right)}{\epsilon^{2}+2 \epsilon\left[q \rho^{2}(1+r)-r C_{1}\right]+\left[1+\left(q^{2}-1\right) \rho^{2}(1+r)-r C_{2}\right]}\right\} .
$$

In Eq. (43a) we have defined

$$
\begin{aligned}
& r \equiv\left(\sigma_{P} / \sigma_{Q}\right)_{0}, \\
& C_{1} \equiv q \operatorname{Re}\langle\alpha\rangle_{P}-\operatorname{Im}\langle\alpha\rangle_{P}, \\
& C_{2} \equiv\left(q^{2}+1\right)\left\langle|\alpha|^{2}\right\rangle_{P}-2 q \operatorname{Im}\langle\alpha\rangle_{P}-2 \operatorname{Re}\langle\alpha\rangle_{P} .
\end{aligned}
$$

Thus Eq. (43a) for the branching ratio within a resonance depends on only two independent linear combinations, $C_{1}$ and $C_{2}$, of the three parameters $\left\langle|\alpha|^{2}\right\rangle_{p}, \operatorname{Re}\langle\alpha\rangle_{p}$, and $\operatorname{Im}\langle\alpha\rangle_{P}$. Given $r, q, \rho^{2}$ and $\Gamma$, one may determine $C_{1}$ and $C_{2}$ by fitting Eq. (43a) to experimental data on the branching ratio within a resonance.

\section{B. Bounds on the parameters}

Equations (28) and (43a) represent a ratio, of cross sections. Hence they must always be nonnegative. Thus in each case we must require the roots of the quadratic polynomials in $\epsilon$, in both nume rator and denominator, to be imaginary. The requirement that these roots be imaginary implies, for the polynomial in the numerator in Eq. (28), that

$$
\begin{aligned}
& 1-2 q \operatorname{Im}\langle\alpha\rangle_{P}-2 \operatorname{Re}\langle\alpha\rangle_{P}+\left(q^{2}+1\right)\left\langle|\alpha|{ }^{2}\right\rangle_{P} \\
& \geq\left(q \operatorname{Re}\langle\alpha\rangle_{P}-\operatorname{Im}\langle\alpha\rangle_{P}\right)^{2},
\end{aligned}
$$

with a similar requirement for the polynomial in the denominator. Equation (44) can be shown to be satisfied provided the $\alpha$ parameters satisfy the
Schwartz inequality

$$
\left\langle|\alpha|^{2}\right\rangle_{P} \geqslant\left(\operatorname{Re}\langle\alpha\rangle_{P}\right)^{2}+\left(\operatorname{Im}\langle\alpha\rangle_{P}\right)^{2} .
$$

The proof proceeds by subtracting the right-hand side of Eq. (44) from both sides of Eq. (44), substituting the right-hand side of Eq. (45) for $\left\langle|\alpha|{ }^{2}\right\rangle_{P}$, and rearranging terms to find

$$
\left[q \operatorname{Im}\langle\alpha\rangle_{P}+\left(\operatorname{Re}\langle\alpha\rangle_{P}-1\right)\right]^{2} \geqslant 0,
$$

which is obviously true. From the definitions in Eqs. (30) and (31) it is clear that Eq. (45) is true. In addition, Eq. (33) and the fact that $\chi \leqslant 1$ implies that

$$
\rho^{2}(1+1 / r) \geqslant\left\langle|\alpha|^{2}\right\rangle_{P} .
$$

These bounds, in Eqs. (45) and (47), must be satisfied by the three independent parameters $\left\langle|\alpha|^{2}\right\rangle_{P}, \operatorname{Re}\langle\alpha\rangle_{P}$, and $\operatorname{Im}\langle\alpha\rangle_{P}$. Similar bounds may be obtained on the parameters $\left\langle|\alpha|^{2}\right\rangle_{Q}$, $\operatorname{Re}\langle\alpha\rangle_{Q}$, and $\operatorname{Im}\langle\alpha\rangle_{Q}$, namely,

$$
\begin{aligned}
& \left\langle.|\alpha|^{2}\right\rangle_{Q} \geqslant\left(\operatorname{Re}\langle\alpha\rangle_{Q}\right)^{2}+\left(\operatorname{Im}\langle\alpha\rangle_{Q}\right)^{2}, \\
& \rho^{2}(1+\gamma) \geqslant\left\langle|\alpha|^{2}\right\rangle_{Q} .
\end{aligned}
$$

Applying the same restrictions on the roots of the polynomials in Eq. (43a) gives two bounds on the parameters $C_{1}$ and $C_{2}$ :

$$
\begin{aligned}
& 1+C_{2} \geqslant C_{1}{ }^{2}, \\
& 1+\left(q^{2}-1\right) \rho^{2}(1+r)-r C_{2} \geqslant\left[q \rho^{2}(1+r)-r C_{1}\right]^{2} .
\end{aligned}
$$


In fitting Eq. (43a) to experimental data, the inequalities in Eqs. (50) and (51) must be enforced or spurious results will be obtained. The inequalities may be satisfied automatically by changing independent variables from $C_{1}$ and $C_{2}$ to two fictitious angles $\theta$ and $\phi$ as following:

$$
\begin{aligned}
& C_{1} \equiv \frac{R}{(1+r)^{1 / 2}} \sin \theta \sin \phi+q \rho^{2}, \\
& C_{2} \equiv-1+R^{2} \sin ^{2} \theta \cos ^{2} \phi+\left(C_{1}\right)^{2},
\end{aligned}
$$

where for compactness we have defined

$$
R^{2} \equiv(1+1 / \gamma)\left[1+\left(q^{2}-1\right) \rho^{2}-q^{2} \rho^{4}\right]
$$

Note that $R^{2} \geqslant 0$ since $\rho^{2} \leqslant 1$.

An additional bound relating $\chi$ and $\xi$ may be obtained by substituting Eqs. (33) and (37) into Eq. (45) to get

$$
\chi \geqslant \rho^{2}(1+1 / r)|\xi|^{2} .
$$

Similarly, substituting Eqs. (35) and (39) into Eq. (48) gives

$$
1-\chi \geqslant \rho^{2}(1+r)|1-\xi|^{2} \text {. }
$$

\section{Maximum and minimum of the branching ratio}

Equations (28) and (43a) may be written in the form

$$
\frac{\sigma_{P}}{\sigma_{Q}}=\left(\frac{\sigma_{P}}{\sigma_{Q}}\right)_{0}\left(1+\frac{A_{1} \epsilon+A_{0}}{\epsilon^{2}+B_{1} \epsilon+B_{0}}\right),
$$

where, for example, in the case of Eq. (28) the coefficients are given by

$$
\begin{aligned}
& B_{1} \equiv 2\left(q \operatorname{Re}\langle\alpha\rangle_{Q}-\operatorname{Im}\langle\alpha\rangle_{Q}\right), \\
& B_{0} \equiv 1-2 q \operatorname{Im}\langle\alpha\rangle_{Q}-2 \operatorname{Re}\langle\alpha\rangle_{Q}+\left(q^{2}+1\right)\left\langle|\alpha|^{2}\right\rangle_{Q},
\end{aligned}
$$

$$
\begin{aligned}
A_{1} \equiv & 2\left(q \operatorname{Re}\langle\alpha\rangle_{P}-\operatorname{Im}\langle\alpha\rangle_{P}\right)-B_{1}, \\
A_{0} \equiv & 1-2 q \operatorname{Im}\langle\alpha\rangle_{P}-2 \operatorname{Re}\langle\alpha\rangle_{P} \\
& +\left(q^{2}+1\right)\left\langle|\alpha|^{2}\right\rangle_{P}-B_{0} .
\end{aligned}
$$

The maximum and minimum values of the function

$$
f(\epsilon) \equiv \frac{A_{1} \epsilon+A_{0}}{\epsilon^{2}+B_{1} \epsilon+B_{0}}
$$

then occur at $\epsilon_{+}$and $\epsilon_{-}$, respectively, where

$$
\epsilon_{ \pm}=\left(1 / A_{1}\right)\left\{-A_{0} \mp\left[A_{0}^{2}+A_{1}\left(A_{1} B_{0}-A_{0} B_{1}\right)\right]^{1 / 2}\right\},
$$

and have the values

$$
f\left(\epsilon_{ \pm}\right)=\frac{\mp A_{1}^{2}}{2\left[A_{0}^{2}+A_{1}\left(A_{1} B_{0}-A_{0} B_{1}\right)\right]^{1 / 2} \mp\left(2 A_{0}-A_{1} B_{1}\right)} .
$$

\section{SUMMARY}

In this paper we have determined, in Eqs. (25) and (26), the behavior of an individual observablechannel dipole-matrix element and its absolute square in terms of a new complex parameter $\alpha(\mu E)$, defined in Eq. (24), and the Fano profile parameters $q$ and $\Gamma$. In Sec. IV this result is used to obtain an expression for the photoelectron branching ratio in the neighborhood of a resonance. We have in mind closed-shell atoms where photoionization of an $n p^{6}, n d^{10}, n f^{14}$, etc., subshell results in two groups of photoelectrons, each having a different energy corresponding to the two fine-structure levels of the residual ion. These two groups of photoelectrons, indicated by $P$ and $Q$, have a branching ratio across a resonance that is given by Eq. (28) in terms of four different averages [cf. Eqs. (30) and (31)] of the $\alpha(\mu E)$ parameters: $\langle\alpha\rangle_{P},\left\langle|\alpha|^{2}\right\rangle_{P},\langle\alpha\rangle_{Q}$, and $\left\langle|\alpha|^{2}\right\rangle_{Q}$ where $\langle\alpha\rangle_{P}$ and $\langle\alpha\rangle_{Q}$ are complex and thus have two components.

Relations between $\left\langle|\alpha|^{2}\right\rangle_{P}$ and $\left\langle|\alpha|^{2}\right\rangle_{Q}$, and between the real and imaginary parts of $\langle\alpha\rangle_{P}$ and $\langle\alpha\rangle_{Q}$ in Eqs. (36), (41), and (42), reduce the number of new parameters needed to calculate the branching ratio from six to three provided the profile parameters $q, \Gamma$ and $\rho^{2}$ and the branching ratio outside the resonance, $\left(\sigma_{p} / \sigma_{Q}\right)_{0}$, are available from other experiments. At present these three independent parameters must be calculated-they cannot be obtained from any experimental data. Thus in order to predict branching ratios within a resonance, one must calculate $\left\langle|\alpha|^{2}\right\rangle_{P}$ and the complex parameter $\langle\alpha\rangle_{P}$. One needs also the profile parameters $q, \rho^{2}$, and $\Gamma$ for the resonance, which may be either obtained from experimental data on the resonance profile or else calculated using Eqs. (5c), (22), and (32). Lastly, one needs the branching ratio outside the resonance, which may be obtained from experimental data on partial cross sections or calculated using Eq. (29).

Alternatively, one may fit experimental data to Eq. (43a), as was done in Ref. 15 , to obtain the two parameters $C_{1}$ and $C_{2}$ defined in Eqs. (43c) and (43d). These parameters are two independent linear combinations of $\left\langle|\alpha|^{2}\right\rangle_{P}, \operatorname{Re}\langle\alpha\rangle_{P}$, and $\operatorname{Im}\langle\alpha\rangle_{p}$. They must satisfy the inequalities in Eqs. (50) and (51), which is easily done by changing variables according to Eq. (52).

While Sec. IV of this paper considers a particular kind of branching ratio in the neighborhood of a resonance (i.e., one where $N$ final-state channels may be split into only two groups corresponding to two groups of ejected electrons), we note that other kinds of branching ratios may be treated easily. One starts from the general result in Eq. (26) for 


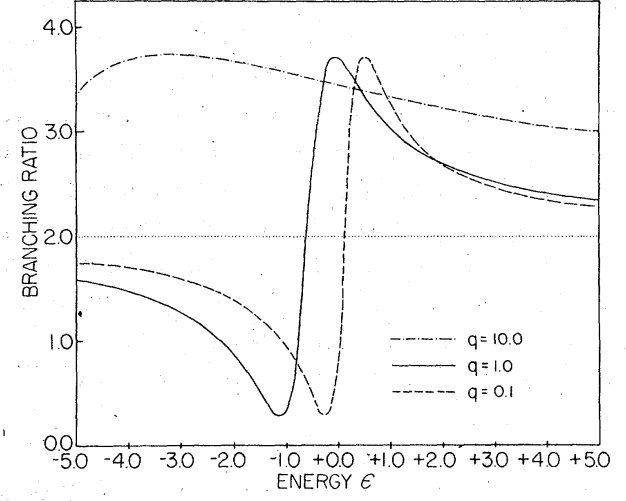

FIG. 1. Behavior of the branching ratio as a function of the reduced energy $\epsilon$ for three values of the parameter $q$. For all curves $r=2.0, \rho^{2}=0.9$, and $\theta=\phi=-\pi / 4$ [cf. Eqs. (43) and (52)]. The dotted curve indicates the value of $r$, the branching ratio away from resonance.

a channel cross section in the neighborhood of a resonance. One then proceeds in a fashion analogous to that in Sec. IV.

\section{DISCUSSION}

A. Illustrations of branching ratio behavior within resonances

Figures 1-3 show various plots of the branchingratio formula presented in Eq. (43a). Equation (52) is used to represent $C_{1}$ and $C_{2}$ in terms of the fictitious angles $\theta$ and $\phi$ so that the boundary conditions on $C_{1}$ and $C_{2}$ are automatically satisfied. Thus the figures show the branching ratio as a function of $\epsilon$ and of the parameters $q, \rho^{2}, r$ $\equiv\left(\sigma_{P} / \sigma_{Q}\right)_{0}, \theta$, and $\phi$. The values of the parameters were chosen to exhibit a variety of possible shapes for the branching-ratio curve and to illustrate the dependence of the branching ratio on those parameters which are characteristic of the resonance

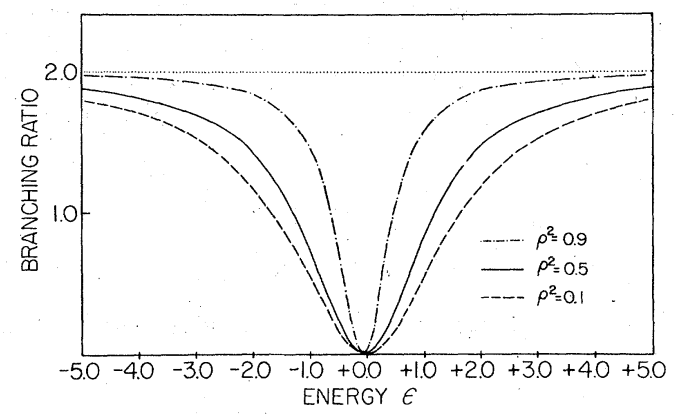

FIG. 2. Behavior of the branching ratio as a function of the reduced energy for three values of the parameter $\rho^{2}$. For all curves, $r=2.0, q=0.1$, and $\theta=\phi=0.0$ [cf. Eqs. (43) and (52)]. The dotted curve indicates the value of $r$, the branching ratio away from resonance. (i.e., $q, \rho^{2}, \theta$, and $\phi$ ). An additional shape for the branching ratio, different from those shown in Figs. 1-3, is given in Ref. 15 using parameter values obtained for a particular resonance. In general, of course, the branching ratio always has a single minimum and a single maximum in the neighborhood of a resonance [cf. Eq. (58)].

In Fig. 1 we have chosen $r=2.0, \rho^{2}=0.9$, and $\theta=\phi=-\pi / 4$, and illustrate how the branching ratio changes as $q$ assumes the three values $10.0,1.0$, and 0.1 . We see from the figure that the maxima and minima seem to be independent of the value of $q$. (For $q=10.0$, the minimum occurs off the scale of the figure.) However, the energy range over which the branching ratio oscillates (i.e., the "width") narrows as $q$ decreases. Furthermore, the positions of the maximum and minimum approach $\epsilon=0.0$ as $q$ decreases.

In Fig. 2 we have chosen $r=2.0, q=0.1$, and $\theta=\phi=0.0$, and illustrate how the branching ratio changes as $\rho^{2}$ assumes the three values $0.9,0.5$, and 0.1 . Note that for $q=0.1$ the autoionization profile is of the window type and fairly symmetric with respect to $\epsilon=0.0 .^{12}$ This symmetry is mirrored in Fig. 2 by the branching ratio. More remarkable is the fact that whereas the total photoionization cross section goes to zero only for $\rho^{2}=1.0,{ }^{13}$ we find that for the parameters chosen for Fig. 2 the branching ratio goes to zero for all three values of $\rho^{2}$, none of which are equal to 1 . This implies either that the partial cross section in the numerator becomes very small or zero within the resonance, or that the partial cross section in the denominator becomes very large, or that perhaps a combination of both effects occurs:

Finally, Fig. 3 shows the dependence of the branching ratio on the parameters $C_{1}$ and $C_{2}$

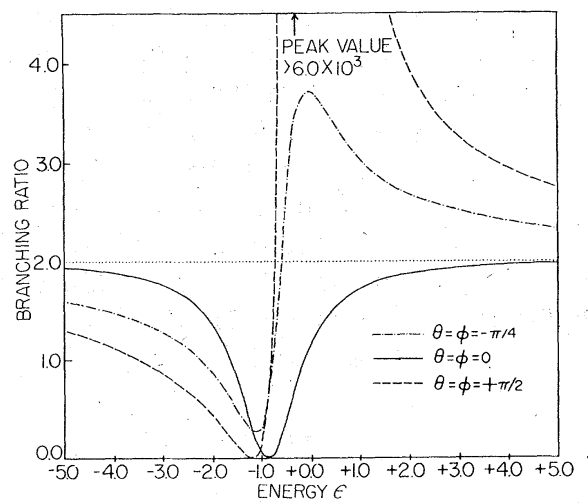

FIG. 3. Behavior of the branching ratio as a function of the reduced energy $\epsilon$ for three values of the parameters $\theta=\phi$. For all curves, $r=2.0, q=1.0$, and $\rho^{2}=0.9$ [cf. Eqs. (43) and (52)]. The dotted curve indicates the value of $r$, the branching ratio away from the resonance. 
through their dependence on the fictitious angles $\theta$ and $\phi$. We have chosen $\theta=\phi, r=2, \rho^{2}=0.9$, and $q=1.0$. We see that for $\theta=\phi=0$ there is a fairly symmetric branching ratio, but that for nonzero values of these angles there is an asymmetric branching ratio. In particular, notice that the dashed curve, for which $\theta=\phi=\pi / 2$, has a minimum of zero and a very large maximum. This indicates either that one of the two partial cross sections oscillates between zero and infinity within the resonance, or that each partial cross section goes to zero at different energies within the resonance, or even perhaps that each partial cross section goes to infinity at different energies within the resonance.

\section{B. Variation of the branching ratio along a Rydberg series}

If the discrete resonance state $\phi$ is the $n$th member $\phi_{n}$ of a Rydberg series, then we may ask how the branching ratio varies from one member of the series to another, or equivalently, how the parameters $q, \rho^{2}, \Gamma$, and $\alpha(\mu E)$ vary from one member of a Rydberg series to another. Quantum-defect theory provides an answer ${ }^{27}$ : The wave function $\phi_{n}$ may be represented as $\phi_{n}=N_{n} \bar{\phi}_{n}$ where $N_{n}$ is an energy-dependent (i.e., $n$-dependent) normalization factor and $\bar{\phi}_{n}$ is the reduced wave function, which is independent of energy for small radii. Thus, provided the matrix elements involving $\phi_{n}$ obtain their major contribution from small radii, the energy dependence of these matrix elements is given by $N_{n}$. Using these arguments, it can be shown that $q$ and $\rho^{2}$ are the same for all members of a Rydberg series and that $\Gamma$ is proportional to $N_{n}^{2}{ }^{13}$ which in turn is proportional to $\left(n^{*}\right)^{-3},{ }^{28}$ where $n^{*}$ is the effective quantum number. In a similar way, examination of Eq. (24) shows that the factor $N_{n}^{2}$ arising from the matrix element in the numerator is cancelled by the factor $N_{n}^{2}$ arising from $\Gamma(E)$ in the denominator. Hence $\alpha(\mu E)$ and its averages are the same for all members of a Rydberg series. Furthermore, examination of Eqs. (43c) and (43d) shows that the parameters $C_{1}$ and $C_{2}$ are the same for all members of a Rydberg series. Thus the branching ratio is expected to have the same behavior for all members of a Rydberg series, except for the narrowing due to the decreasing linewidth $\Gamma$.

\section{Relation to other work}

Dill ${ }^{16}$ has developed the theory for the variation of the angular distribution asymmetry parameter $\beta$ within an autoionizing resonance using a very general framework: the angular-momentumtransfer expansion for $\beta$. The dipole-matrix ele- ments and phase shifts needed to compute $\beta$ are obtained for a particular case by means of the multichannel quantum-defect theory. "In this theory the autoionizing resonance is treated as a member of a closed channel and not as an isolated resonance as in this paper. To compensate for this greater generality, however, a much larger number of experimental data have to be analyzed to obtain the quantum-defect-theory parameters needed for the calculation. No explicit relation is made to the profile parameters $q, \rho^{2}$, and $\Gamma$ of the resonance.

Kabachnik and Sazhina, ${ }^{20}$ on the other hand, develop the theory for the variation of $\beta$ and of the photoelectron spin polarization within a resonance in a way that is very similar to that used in this paper. That is, they regard the resonance as isolated and make use of the $q$ parameter for the resonance. However, they make the additional assumption that the prediagonalized states $|i \epsilon\rangle$ are identical to the asymptotically observable states $|\mu \epsilon\rangle$, an approximation which is not a good one for heavier atoms. However, this approximation does not affect the form of the dipole-matrix elements within a resonance. In particular, our expression for the dipole-matrix element, Eq. (25), reduces to the complex conjugate of Eq. (45) in Ref. 20 in the limit of no first-order interchannel interactions. This reduction proceeds as follows: The limit of no first-order interchannel interactions implies that the matrix $\tilde{T}_{i \mu}(E)$ becomes the unit matrix $\delta_{i \mu}$. Equation (17) then gives

$$
\Omega_{\lambda_{\mu}}(E)-\tilde{U}_{\lambda \mu}(E) \exp \left(-i \eta_{\mu}\right) .
$$

Substituting Eq. (59) in Eq. (24) gives

$$
\begin{aligned}
\alpha(\mu E) & \left\langle\psi_{0}|\overrightarrow{\mathrm{r}}| \mu E-\right\rangle_{0} \\
& \rightarrow \sum_{\mu=1}^{N}\left\langle\psi_{0}|\overrightarrow{\mathrm{r}}| \mu E\right\rangle U_{\mu_{1}}(E) \tilde{U}_{1 \mu}(E) \exp \left(-i \eta_{\mu}\right) .
\end{aligned}
$$

Comparison now of Eqs. (25) and (60) of this paper with Eqs. (44) and (45) of Ref. 20 reveals that in the limit that the states $|\mu \epsilon\rangle$ and $|i \epsilon\rangle$ are identical (i.e., in the limit of no first-order interchannel interactions), our parameters reduce to those of Ref. 20 as follows:

$$
\begin{aligned}
& \alpha(\mu E)\left\langle\psi_{0}|\overrightarrow{\mathbf{r}}| \mu E-\right\rangle_{0} \rightarrow R_{\mu}^{*} \exp \left(-i \delta_{\mu}\right), \\
& \left\langle\psi_{0}|\overrightarrow{\mathbf{r}}| \mu E-\right\rangle_{0} \rightarrow\left(R_{\mu}^{*}+r_{\mu}^{*}\right) \exp \left(-i \delta_{\mu}\right),
\end{aligned}
$$

where $R_{\mu}, r_{\mu}$, and $\delta_{\mu}$ are parameters defined in Ref. 20.

$$
\begin{aligned}
& \text { D. Complete experimental determination } \\
& \text { of the interaction parameters }
\end{aligned}
$$

In the neighborhood of a particular resonance having known profile parameters, the outcome of 
a particular photoelectron measurement in the neighborhood of the resonance is dependent on the complex dipole-matrix elements in Eq. (25). Conversely, it may be possible to do a number of experimental measurements that would completely determine the dipole-matrix elements in Eq. (25). Such a complete set of measurements away from the resonance would determine the dipole-matrix elements $\left\langle\psi_{0}|\vec{r}| \mu E-\right\rangle_{0}$; the complete set of measurements done within the resonance would then determine the $\alpha(\mu E)$ parameters.

As an example, consider the photoionization of the outer $p^{6}$ subshell of the rare gases. There are five continuum channels $\mu$. Since $\alpha(\mu E)$ is complex, there are ten parameters to determine. Equations (36), (41), and (42) give three relations for these parameters in terms of the profile parameter $\rho^{2}$ and the ${ }^{2} P_{3 / 2}:{ }^{2} P_{1 / 2}$ photoelectron branching ratio outside the resonance. Measure- ment of the branching ratio within the resonance determines $C_{1}$ and $C_{2}$, which give two more relations for the $\alpha(\mu E)$ parameters [cf. Eqs. (43c) and (43d)]. Thus only five more relations are needed. Kabachnik and Sazhina ${ }^{20}$ show that the photoelectron angular distribution is determined by three parameters within a resonance and that the photoelectron spin polarization is determined by a different set of three parameters within a resonance. These parameters, which involve sums of products of dipole-matrix elements of the form of Eq. (25), thus give additional relations for the parameters $\alpha(\mu E)$. In the rare-gas case considered here, measurement of either the spin polarization or the angular distribution for each of the two energy groups of photoelectrons within the resonance would thus determine six parameters, giving one more relation than needed to completely determine the $\alpha(\mu E)$ parameters.
*Supported in part by the U.S. Energy Research and Development Administration.

$\uparrow$ Work supported in part by the Alfred P. Sloan foundation.

${ }^{1}$ See J. A. R. Samson, J. L. Gardner, and A. F. Starace, Phys. Rev. A 12, 1459 (1975) and references therein to earlier work.

${ }^{2}$ J. L. Dehmer and J. Berkowitz, Phys. Rev. A 10, 484 (1974).

${ }^{3}$ W. C. Lineberger and B. W. Woodward, Phys. Rev. Lett. 25, 424 (1970).

${ }^{4}$ H. Hotop, T. A. Patterson, and W. C. Lineberger, Phys. Rev. A 8,762 (1973).

${ }^{5}$ K. T. Lu, Phys. Rev. A $\underline{4}, 579$ (1971).

${ }^{6}$ U. Fano, Comments At. Mol. Phys. 2, 171 (1971).

${ }^{7}$ A. R. P. Rau and U. Fano, Phys. Rev. A $\underline{4}, 1751$ (1971).

${ }^{8}$ C. M. Lee and K. T. Lu, Phys. Rev. A 8, 1241 (1973).

${ }^{9}$ T. E. H. Walker, J. Berkowitz, J. L. Dehmer, and J. T. Waber, Phys. Rev. Lett, 31, 678 (1973).

${ }^{10}$ T. E. H. Walker and J. T. Waber, J. Phys. B 7,674 (1974).

${ }^{11}$ A. R. P. Rau, in Electron and Photon Interactions with Atoms, edited by H. Kleinpoppen and M. R. C. McDowell (Plenum, New York, 1976), p. 141.

${ }^{12}$ U. Fano, Phys. Rev. 124, 1866 (1961).

${ }^{13}$ U. Fano and J. W. Cooper, Phys. Rev. 137, A1364 (1965).

${ }^{14} \mathrm{U}$. Fano and J. W. Cooper, Rev. Mod. Phys. $\underline{40}, 441$ (1968), Sec. 8.1.

${ }^{15}$ P. C. Kemeny, J. A. R. Sams on, and A. F. Starace, J. Phys. B 10, L201 (1977).

${ }^{16}$ D. Dill, Phys. Rev. A 7, 1976 (1973).

${ }^{17}$ J. A. R. Sams on and J. L. Gardner, Phys. Rev. Lett.
31, 1327 (1973).

${ }^{18}$ A. F. Starace, Bull. Am. Phys. Soc. 19, 1203 (1974).

${ }^{19} \mathrm{~A}$. F. Starace, in Abstracts of the Fifth International Conference on Atomic Physics, Berkeley, Calif., 1976 (unpublished), p. 368.

${ }^{20}$ N. M. Kabachnik and I. P. Sazhina, J. Phys. B 9, 1681 (1976).

${ }^{21}$ A. F. Starace, Phys. Rev. B 5, 1773 (1972), Sec. П.

${ }^{22} \mathrm{E}$. Kreyszig, Advanced Engineering Mathematics, 3rd ed. (Wiley, New York, 1972), p. 257.

${ }^{23} \mathrm{Calculation}$ of the coefficients $\chi_{\lambda}^{(\mu E-)}$ is given for a very similar problem in Eqs. (39)-(42) of Ref. 21. In Ref. 21 the channels $\mu$ in this paper are denoted by the double index $(\mu, \sigma)$; the prediagonalized states $i$ in this paper are denoted by $\Gamma$; the eigenchannels $\lambda$ in this paper are denoted by $\Lambda$, and since Ref. 21 considers the interaction of several discrete resonances with $N$ continua, each eigenstate $\Lambda$ in Ref. 21, has a characteristic eigenphase shift $\phi_{\Lambda}$ rather than only one, as in this paper. Note that on the left side of Eq. (41) in Ref. 21, a factor $\bar{U}_{\Gamma, \Lambda}(E)$ is missing and $l$ should be replaced by "exp."

${ }^{24}$ See Eq. (2.8) of Ref. 13.

${ }^{25}$ See Eqs. (2.3) and (2.14) of Ref. 14.

${ }^{26}$ See Eq. (2.12) of Reî. 13. Note that in summing over all $N$ channels it does not matter whether we sum over the set of prediagonalized continua $|j E\rangle$, as in Eq. (2.12) of Ref. 13, or whether we use the set of observable channels $\mu$ as in Eq. (32) of this paper.

${ }^{27}$ A. F. Starace, in Photoionization and Other Probes of Many Electron Interactions, edited by F. J. Wuilleumier (Plenum, New York, 1976), pp. 395-406.

${ }^{28}$ J. L. Dehmer and U. Fano, Phys. Rev. A $\underline{2}, 304$ (1970). 